\title{
The effect of COVID-19 and rise of telehealth on end-of-life conversations
}

\section{cambridge.org/pax}

\section{Essay/Personal Reflection}

*Simran Kripalani and Klaus Danjolli are co-authors and both contributed equally to this piece.

Cite this article: Kripalani S, Danjolli K, McGeehan JF (2022). The effect of COVID-19 and rise of telehealth on end-of-life conversations. Palliative and Supportive Care 20, 144-145. https://doi.org/10.1017/ S1478951520001418

Received: 1 December 2020

Accepted: 18 December 2020

\section{Author for correspondence:}

Simran Kripalani, MS-3, Cooper Medical School of Rowan University, 401 Broadway, Camden, NJ 08103, USA. E-mail: kripal36@rowan.edu

(C) The Author(s), 2021. Published by Cambridge University Press. This is an Open Access article, distributed under the terms of the Creative Commons Attribution licence (http://creativecommons.org/licenses/by/4.0/), which permits unrestricted re-use, distribution, and reproduction in any medium, provided the original work is properly cited.

\section{CAMBRIDGE} UNIVERSITY PRESS
Simran Kripalani, B.s. ${ }^{1, \star}\left(\mathbb{D}\right.$, Klaus Danjolli, B.A. ${ }^{1, \star}$ and John F. McGeehan, M.D. ${ }^{1,2}$

${ }^{1}$ Cooper Medical School of Rowan University, Camden, NJ and ${ }^{2}$ Cooper University Hospital, Camden, NJ

The coronavirus (COVID-19) pandemic has caused a majority of countries to take measures in controlling the spread of this contagious virus. These measures have ranged from limiting gatherings and enforcing social distancing to complete lockdowns with government surveillance. This has, in turn, changed the way many people operate daily. In an effort to limit transmission of COVID-19, many governments and employers have decided to instate work-from-home policies. Because many patients with certain disease processes and conditions are in an immunocompromised state, they are advised to limit their exposure in high-risk settings so that they do not contract COVID-19. With this, healthcare facilities and providers have offered certain services through virtual platforms, such as telehealth. Recently, scientists showed that urgent care telemedicine visits at a large healthcare system in New York, the epicenter of the pandemic, reported a $683 \%$ increase in telemedicine visits just from March to April 2020 (Abbott et al., 2020). With this perspicuous rise in telehealth visits comes an increasingly urgent question: how does this affect end-of-life (EOL) conversations between patient and provider?

At baseline, EOL conversations are already difficult discussions. They often include serious dialogue about prognosis, support systems, future intentions and interventions, healthcare goals, and treatment plans. Due to the magnitude of COVID-19, we must ensure that the nature and integrity of these conversations should not be compromised via telemedicine. Providers should also have these online EOL conversations by learning how to discuss goals of care in EOL discussions (Hewitt et al., 2010). With the second wave of the pandemic on the horizon, and the potential for this state to become our "new normal," EOL conversations may not include a vital component of its character.

For many patients, the dying process is a journey with their loved ones, friends, and healthcare team. They have conversations with various physicians and nurses in different specialties, oftentimes together in the form of a family meeting. Patients experiencing this are able to talk to behavioral medicine, psychiatrists, palliative and hospice care, and pastoral care or hospital chaplains. The presence of one unified team in a central location is undeniably important when it comes to conversations such as family meetings, which are further compromised due to pandemic visitor restrictions. Continual emphasis on these meetings is crucial, as they show commitment to the patient's care, importance on the patient's outcomes, and stresses the gravity of the situation and decisions to be made. The collaborative dialogue, collective silence, and raw body language are essential when conveying human emotion. While silence and space can be devoted to the patient using telehealth as well, it is difficult to imagine that the same effect is elicited when your Outlook calendar sends you notifications about your next patient and the meetings you missed because you forgot to press the "dismiss" button.

The vital component from the EOL meetings conducted over telemedicine is the physical and emotional presence of your providers and loved ones in the room with you. Intimacy is inherently woven into the fabric of palliative care. Through telemedicine visits, this essence is distinctly erased. Researchers have found that, although there are no communicative differences between telemedicine and in-person visits, physicians employing telemedicine are $23 \%$ less likely to ask further questions regarding primary concerns brought up by patients (Mann et al., 2020). Therefore, while these conversations will still happen, they may not be conducted with the same depth and breadth as they would in person. Raw emotions may muddle your thoughts, and you may lose the salient takeaways necessary for your mental and physical well-being. Without the analysis of physical body language, telemedicine conversations may not have the same effect for both the patient and provider if they feel less submerged in the conversation. Additionally, the implementation of telemedicine also leaves behind the therapy of our oldest and most personal healing tool: the human touch. In one study, therapeutic treatments involving the use of gentle touch had a $67 \%$ decrease in severe stress levels for cancer patients, and a $31.5 \%$ decrease in those experiencing severe pain (Tieman et al., 2016). We are aware of the importance of safety and public health measures due to a virus that has taken the lives of over 250,000 Americans and over 1 million human beings around the world. By no means do we suggest any measure that can increase the risk of spread, and, therefore, the risk of human suffering. Despite this, we must consider the impact that this 
pandemic has on EOL conversations, as individuals who crave direct human contact in a sacred, allotted space may not be able to do so.

In the midst of a pandemic affecting millions globally, perhaps telemedicine visits can be cherished as an act of love. These visits may be one of the only ways to restore intimacy in the form of a warm, inviting smile or raw tears. Maybe wearing a mask and goggles in-person may cover an appreciable amount of our face, leading to fragmented empathy. While intimacy via telemedicine may not be ideal, it is an undeniable signal to the person on the receiving end that their concerns and fears are being emphatically met, and seen, by someone with compassion. Telehealth may also promote equity in management of palliative care resources for patients, as clinical time per patient or community can be adjusted based on need (Weze et al., 2004).

As medical students and future healthcare professionals, we urge for an adaptation to our curriculum so that we are able to learn how to use telemedicine resources to fulfill even the most complex tasks, such as the discussion of EOL care. We must find a way to make the impersonal personal. People often state that nothing can replace the feeling and connectedness of the human touch. Whether this decision to move to telemedicine is based on the ubiquitous presence of technology in our society or dire necessity in the setting of a pandemic, only time will tell if a person's touch is best suited on the patient's shoulder or the "end call" button on their cell phone.

\section{References}

Abbott J, Johnson D and Wynia M (2020) Ensuring adequate palliative and hospice care during COVID-19 surges. JAMA. doi:10.1001/ jama.2020.16843

Hewitt H, Gafaranga J and McKinstry B (2010) Comparison of face-to-face and telephone consultations in primary care: Qualitative analysis. British Journal of General Practice 60(574), e201-e212. doi:10.3399/bjgp10X501831

Mann DM, Chen J, Chunara R, et al. (2020) COVID-19 transforms health care through telemedicine: Evidence from the field. Journal of the American Medical Informatics Association 27(7), 1132-1135. doi:10.1093/ jamia/ocaa072

Tieman JJ, Swetenham K, Morgan DD, et al. (2016) Using telehealth to support end of life care in the community: A feasibility study. BMC Palliative Care 15. doi:10.1186/s12904-016-0167-7

Weze C, Leathard HL, Grange J, et al. (2004) Evaluation of healing by gentle touch in 35 clients with cancer. European Journal of Oncology Nursing 8(1), 40-49. doi:10.1016/j.ejon.2003.10.004 